\title{
Launching Annals of Liver Transplantation: First small steps toward remarkable academic achievements in liver transplantation
}

\author{
Shin Hwang, Editor-in-chief, Annals of Liver Transplantation
}

This year is the 22th anniversary of the Korean Liver Transplantation Society (KLTS). During the past 32 years following the first liver transplantation in Korea, remarkable advances have been made in clinical and research fields of liver transplantation, especially in living donor liver transplantation. Korea has become one of leading countries in the clinical field of liver transplantation. The KLTS represents clinical and academic aspects of liver transplantation in Korea.

For further development of KLTS, Annals of Liver Transplantation (ALT), the official journal of the KLTS, was launched. This is the first issue of ALT. ALT is an international, peer-reviewed open access journal. This journal pursues its advancement through original articles, reviews, case reports, editorials, and letters to the editor. The journal is for clinicians and scientists in liver transplantation. It is also for those in other fields who are interested in liver transplantation.

The aim of ALT is to make contribution to saving lives of patients undergoing liver transplantation through active communication and exchange of study information on liver transplantation and provision of education and training on liver diseases. The ALT serves as a platform for debate and reassessment, a trigger of innovation, and a major pedestal for promoting understanding, improving outcomes, and advancing knowledge and techniques for liver transplantation.

Regarding liver transplantation in Korea and Asian countries, problems and viewpoints are different from those of Western countries. Ethical and legislative problems are more serious. The situation of organ donation and level of research are also different from those in other regions. Severe donor shortage has led to marked development in living donor liver transplantation. Thus, editorial members of ALT will make more efforts to reflect characteristics of our regional academy.

Recently, commercialism of scientific journals has increased to the extent that it has increased the financial burden for authors. ALT will be a good journal at the Science Citation Index level without imposing undue financial burden to authors. One of the most important aspects of publication in a scientific journal is easy and rapid communication. Thus, ALT will provide easy accessibility and convenience for authors and readers.

The ALT is starting with small steps now. These small steps will lead to remarkable achievements that members of the KLTS and International Liver Transplantation Society will be proud of in the near future. 